\title{
Primary outgrowth cultures are a reliable source of human pancreatic stellate cells
}

\author{
Song Han ${ }^{1,4}$, Daniel Delitto ${ }^{1,4}$, Dongyu Zhang ${ }^{1}$, Heather L Sorenson ${ }^{2}$, George A Sarosi ${ }^{1,3}$, Ryan M Thomas ${ }^{1,3}$, \\ Kevin E Behrns ${ }^{1}$, Shannon M Wallet ${ }^{2}$, Jose G Trevino ${ }^{1}$ and Steven J Hughes ${ }^{1}$
}

Recent advances demonstrate a critical yet poorly understood role for the pancreatic stellate cell (PSC) in the pathogenesis of chronic pancreatitis (CP) and pancreatic cancer (PC). Progress in this area has been hampered by the availability, fidelity, and/or reliability of in vitro models of PSCs. We examined whether outgrowth cultures from human surgical specimens exhibited reproducible phenotypic and functional characteristics of PSCs. PSCs were cultured from surgical specimens of healthy pancreas, CP and PC. Growth dynamics, phenotypic characteristics, soluble mediator secretion profiles and co-culture with PC cells both in vitro and in vivo were assessed. Forty-seven primary cultures were established from 52 attempts, demonstrating universal $a$-smooth muscle actin and glial fibrillary acidic protein but negligible epithelial surface antigen expression. Modification of culture conditions consistently led to cytoplasmic lipid accumulation, suggesting induction of a quiescent phenotype. Secretion of growth factors, chemokines and cytokines did not significantly differ between donor pathologies, but did evolve over time in culture. Co-culture of PSCs with established PC cell lines resulted in significant changes in levels of multiple secreted mediators. Primary PSCs co-inoculated with PC cells in a xenograft model led to augmented tumor growth and metastasis. Therefore, regardless of donor pathology, outgrowth cultures produce PSCs that demonstrate consistent growth and protein secretion properties. Primary cultures from pancreatic surgical specimens, including malignancies, may represent a reliable source of human PSCs.

Laboratory Investigation (2015) 95, 1331-1340; doi:10.1038/labinvest.2015.117; published online 7 September 2015

Evidence continues to accumulate implicating the intense desmoplastic reaction common to both chronic pancreatitis (CP) and pancreatic cancer (PC) is not simply a bystander process from a dynamic inflammatory state, but rather represents a complex interplay within the microenvironment responsible for the initiation and maintenance of key pathologic feedback systems. ${ }^{1}$ Pancreatic stellate cells (PSCs) appear to represent a critical determinant in the development and maintenance of this desmoplastic microenvironment in pancreatic disease states. ${ }^{2-4}$ However, recent investigations have questioned the role of PSCs in cancer. Specifically, depletion of activated PSCs in genetically engineered mouse models of PC has paradoxically demonstrated pro-tumor effects. ${ }^{5,6}$ While disparate from prior observations showing the tumor promoting abilities of activated PSCs, ${ }^{3,7-9}$ these investigations still implicate an interplay between PSCs, other cells within the tumor microenvironment, and the cancer. ${ }^{5,6}$
Given these disparate findings, further investigation into the biology of PSCs in various disease states is essential and necessitates a high degree of experimental standardization. To this effect, an expert panel recently published guidelines regarding PSC isolation, identification, and activation. ${ }^{10}$ Human PSCs are most typically isolated via the outgrowth method, whereby direct culture of minced pancreatic specimens yields activated PSCs with limited replicative potential. ${ }^{1-13}$ This approach remains a vital experimental model strategy for human PSCs as immortalization imparts significant genetic instability. ${ }^{10}$ However, owing to the surgical specialization required and relative infrequency of pancreatic operations, centers investigating PSC biology are often unable to achieve sufficient human specimens to consistently and reliably generate primary cultures. Further, pancreatic resections are performed for a diverse array of pathologies, but most typically are performed for malignancy. Whether the particular pathology necessitating resection imparts physiologic changes

\footnotetext{
'Department of Surgery, College of Medicine, University of Florida Health Science Center, Gainesville, FL, USA; ${ }^{2}$ Department of Periodontology and Oral Biology, College of Dentistry, University of Florida Health Science Center, Gainesville, FL, USA and ${ }^{3}$ Department of Surgery, North Florida/South Georgia Veterans Health System, University of Florida College of Medicine, Gainesville, FL, USA

Correspondence: Dr SJ Hughes, MD, Department of Surgery, University of Florida College of Medicine, Room 6116, Shands Hospital, 1600 SW Archer Road, Gainesville, FL 32610, USA. 
that persist in PSC outgrowth cultures, represents a fundamental scientific question with direct implications for further investigations into PSC biology.

Taken together, these issues highlight the translational value in defining PSC biology with respect to the pathogenesis of pancreatic disease. We report the successful culture and characterization of human PSCs primary cultures from 47 pancreatic specimens, supporting outgrowth cultures as an efficient, high-throughput, reproducible, isolation technique for human PSCs. Our characterization was based on consensus guidelines in a cohort of outgrowths representing a range of pancreatic pathologies. ${ }^{10}$ As PSC biology is often investigated in the context of paracrine signaling leading to inflammation and/or cancer progression, PSC soluble mediator secretion profiles were evaluated in culture with respect to donor pathology and extent of passage. Further, consistent changes in secretion profiles with PC cell co-culture were demonstrated as well as functional augmentation of tumor growth and metastasis of co-inoculated PSC/PC cells in a murine xenograft model.

\section{MATERIALS AND METHODS Cell Lines and Reagents}

Human PC cell lines BxPC3 and PANC-1 were obtained from American Type Culture Collection (Rockville, MD, USA) and were used within 6 months and authenticated by STR analysis. The metastatic PC cell line L3.6pl was derived as previously described. ${ }^{14,15}$ The human fetal primary pancreatic fibroblast (HPF) cell line was purchased from Vitro Biopharma (Golden, CO, USA). Growth characteristics of HPF cells have been validated and publicized by the manufacturer. Cells were maintained in culture with Dulbecco's Modified Eagle's Medium-F12 (DMEM-F12), $10 \%$ fetal bovine serum (FBS) (Atlanta Biologicals, Atlanta, GA, USA), antibiotic antimycotic solution (Fisher Scientific, Waltham, MA, USA) and $5 \% \mathrm{CO}_{2}$ at $37^{\circ} \mathrm{C}$.

\section{Isolation and Culture of Human Pancreatic Stromal Outgrowths}

Primary PSCs were isolated from fresh pancreatic surgical specimens under an institutional review board approved protocol. Informed consent was obtained from all patients preoperatively. Fresh tissues were obtained in complete culture medium on ice within $30 \mathrm{~min}$ of operative resection. Tissue fragments were then dissected into $\sim 1 \mathrm{~mm}^{3}$ pieces and six-eight pieces were placed in each well of a six-well plate pre-coated with $10 \mathrm{mM} \mathrm{CaCl}_{2} / \mathrm{FBS}$. Cells were passaged at a density of $2.5 \times 10^{5}$ cells per $100 \mathrm{~mm}$ dish and grown to $80 \%$ confluence. Cell counts were determined using the TX20 (Bio-Rad Laboratories, Hercules, CA, USA). Population doubling levels (PDL) were calculated using the formula: $\mathrm{PDL}=3.32 \times\left(\log X_{\mathrm{e}}-\log X_{\mathrm{b}}\right)+\mathrm{S} \times X_{\mathrm{b}}$ where $X_{\mathrm{b}}$ and $X_{\mathrm{e}}$ represent cell numbers at the beginning and end of the incubation time.

\section{Flow Cytometry Analysis}

Primary PSCs were fixed, permeabilized, and stained with primary antibodies, including anti- $\alpha$-smooth muscle actin (SMA)-AF488 (R\&D systems, Minneapolis, MN, USA), anti-epithelial surface antigen (ESA)-phycoeryrthrin (PE) (Miltenyi Biotec, San Diego, CA, USA), or anti-glial fibrillary acidic protein (GFAP) (Dako, Carpinteria, CA, USA) with anti-rabbit IgG-AF488 (Invitrogen, Carlsbad, CA, USA). Dual staining for anti-Ki67-FITC (BD Biosciences, San Jose, CA, USA) and anti- $\gamma$-H2A-AF647 (Cell Signaling) was used for assessing senescence. Flow cytometric analysis employed the LSRII flow cytometer and data were analyzed with FACSDiva software (BD Biosciences).

\section{Fluorescence-Activated Cell Sorting}

To further characterize the ESA ${ }^{\mathrm{dim}}$ PSCs, PSCs were labeled with PE conjugated anti-ESA antibody and cell sorting was performed using the FACS Aria-II (BD Biosciences). The PE signal was collected in the FL1 channel through a 575/25 bandpass filter. Cells were displayed in a single-parameter histogram and ESA-PE ${ }^{\mathrm{dim}}$ PSCs were collected in DMEM-F12 with $10 \%$ FBS.

\section{Immunofluorescence}

Cells cultured on glass slides were fixed and permeablized with cold methanol/acetone solution $(1: 1)$ at $-20^{\circ} \mathrm{C}$ for $5 \mathrm{~min}$. Antibodies for specific markers included: anti- $\alpha$-SMA (SigmaAldrich, St. Louis, MO, USA), anti-GFAP (Dako, Carpinteria, CA, USA), and anti-ESA (Abcam, Cambridge, England). Secondary Alexa Fluor 488 conjugated anti-mouse or antirabbit IgG (Life Technologies, Carlsbad, CA, USA) were then applied. Acti-stain 488 was purchased from Cytoskeleton. Imaging was performed using the Olympus IX81-DSU Spinning Disk confocal microscope (Olympus Corporation, Tokyo, Japan).

\section{Co-Culture Model}

Early passage (P2-3) normal-associated stroma (NAS) lines were co-cultured with PC cell lines at a ratio of 10:1 PSC:PC cells for $24 \mathrm{~h}$. Each PC cell line was co-cultured separately with each NAS line. Cells were given $24 \mathrm{~h}$ to adhere before experimentation. Control groups consisted of equivalent cell numbers of either PSCs or PC cells alone.

\section{Analysis of Soluble Mediator Secretion}

Supernatants of cultured PSC, PC cell lines, and co-cultures were collected after $24 \mathrm{~h}$ of serum starvation. Supernatants were then probed for soluble mediators using the Milliplex Premixed 41-Plex Immunology Multiplex Assay (Merck Millipore, Darmstadt, Germany). Concentrations were quantified using a standard curve and five parameter logistics to determine $\mathrm{pg} / \mathrm{ml}$ concentrations. All samples were batch run in duplicate. 

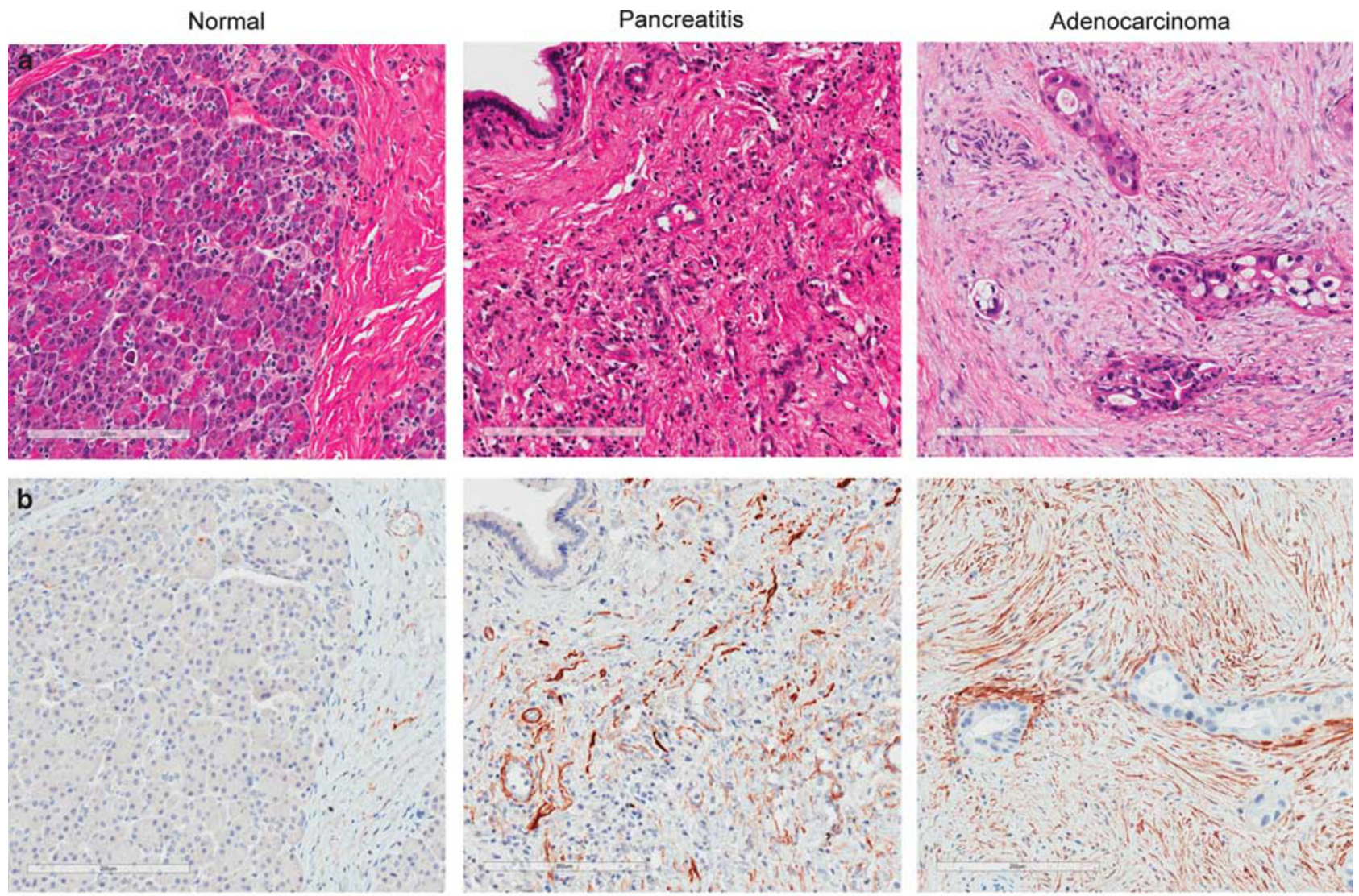

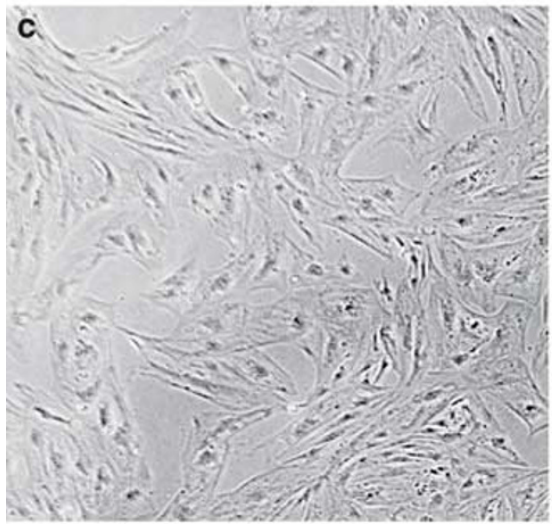

NAS

$3 / 6(50.0 \%)$

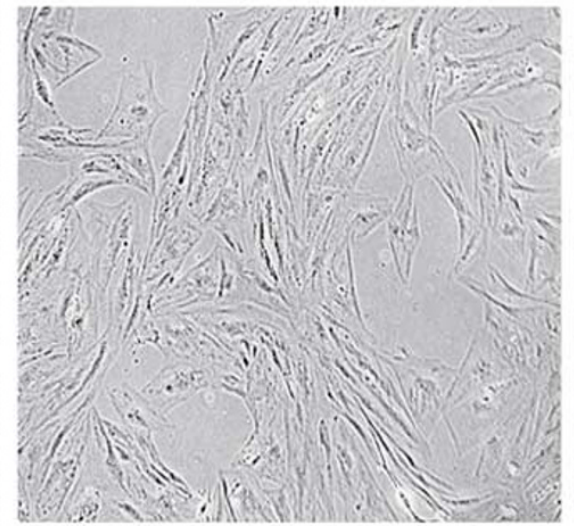

IAS

$7 / 8(87.5 \%)$

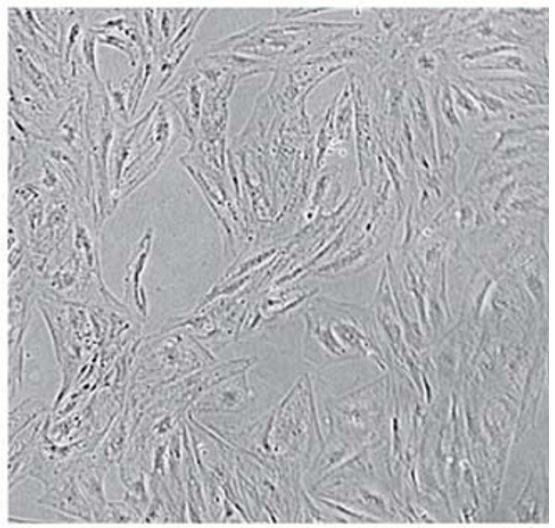

TAS

37/38 (97.4\%)

Figure 1 Culture of stromal outgrowths from human pancreatic tissues. (a) Outgrowth cultures were broadly categorized into three separate donor pathologies: NAS from benign pancreatic disease without histologic evidence of CP, IAS from CP specimens and TAS from pancreatic adenocarcinoma. Diagnoses were confirmed by H\&E examination of immediately adjacent tissue and cross-referenced to clinical pathologic diagnosis. (b) Immunohistochemical staining depicts the presence of stromal $a$-SMA-positive cells in human pancreatic tissues. (c) Phase contrast images of outgrowth morphology are displayed as well as the rate of successful outgrowth culture with respect to donor pathology. Histologic sections and cells in culture are viewed at $\times 10$. CP, chronic pancreatitis; H\&E, hematoxylin and eosin; IAS, inflammation-associated stroma; NAS, normal-associated stroma; $a$-SMA, $a$-smooth muscle actin; TAS, tumor-associated stroma.

\section{Oil Red Staining}

Confluent cells were exposed to adipogenesis differentiation medium (DMEM-F12 medium supplemented with dexamethasone $1 \mu \mathrm{M}$, pantothenate $17 \mu \mathrm{M}$, and human recombinant insulin
$1 \mu \mathrm{M})$ for 3 weeks. ${ }^{16}$ Cells were then fixed in $10 \%$ neutral buffered formaldehyde for $30 \mathrm{~min}$, washed in distilled water and stained using $60 \%$ oil red O (Sigma) solution for $15 \mathrm{~min}$. Images were acquired by the EVOS Cell Imaging System. 


\section{Animal Experiments}

All in vivo studies were approved by the Institutional Animal Care and Use Committee of the University of Florida. Primary tumor-associated stroma (TAS) cells $\left(10^{5}\right.$ cells) were co-injected with luciferase-transfected $\mathrm{L} 3.6 \mathrm{pl}\left(10^{3}\right.$ cells $)$ into the flanks of NOD-SCID mice (Jackson Laboratories, Bar Harbor, ME, USA). Bioluminescence imaging was performed each week and intensities recorded. Animals were euthanized once tumors reached $1.5 \mathrm{~cm}$ in size as measured by calipers. Tumor volume was calculated using the formula $v=x^{2} y / 2$, where $x$ is the minimal and $y$ is the maximal tumor dimension.

\section{Statistical Analysis}

Data were analyzed using the GraphPad Prism version 6.0. Normality was assessed using the Shapiro-Wilk test. Owing to the nonparametric nature of data, the MannWhitney $U$-test was used for comparisons between two groups, and the Kruskal-Wallis test was used for comparisons between three groups. Differences were considered significant at $P<0.05$.

\section{RESULTS}

\section{Stromal Outgrowth Cultures from Pancreatic Surgical Specimens}

Stromal outgrowth cultures were attempted from 51 pancreatic surgical specimens. Pathologies were broadly categorized into TAS from PC specimens, inflammation-associated stroma (IAS) from donors with $\mathrm{CP}$, and NAS from nonmalignant pancreatic tissue without histologic evidence of chronic inflammation or other pathology. The majority of patients in the NAS category underwent pancreatic resection for either benign cystic lesions or duodenal adenomas. Pathologic categorization was confirmed by H\&E analysis of immediately adjacent tissue (Figure 1a) and cross-referenced against final clinical diagnosis for each specimen. Immunohistochemical analysis of paraffin-embedded tissue sections revealed a preponderance of $\alpha$-SMA-positive cells in both pancreatitis and ductal adenocarcinoma samples, while normal pancreatic specimens exhibited a relative scarcity of $\alpha$-SMA-positive cells (Figure 1b).

Within 3-4 weeks of initial culture, identifiable stellateshaped cells began to migrate from central tissue cores, progressively forming a homogenous monolayer (Figure 1c). In this manner, successful outgrowth from $97.4 \%$ (37/38) of PC specimens, $87.5 \%$ (7/8) of CP specimens, and only $50 \%$ (3/6) of normal pancreatic specimens were obtained. Taken together, we generated a total of 47 primary cultures with a success rate of $90.4 \%(47 / 51)$. These data suggest an association between the abundance of $\alpha$-SMA-positive stromal cells present in pancreatic tissue and the likelihood that subsequent outgrowth cultures will be successful.

\section{Outgrowth Cultures Demonstrate Phenotypes Consistent with Activated PSCs}

Despite the pathologic diversity of surgical samples, outgrowth cultures of each specimen yielded morphologically identical fibroblastic, stellate-shaped cells (Figure 1c). Immunofluorescent staining revealed abundant GFAP and $\alpha$-SMA expression, biochemical markers of activated PSCs. In addition, outgrowths stained negative for ESA (Figure 2a). This finding contrasts with immunohistochemical analysis of $\alpha$-SMA expression in normal pancreatic tissue (Figure 1b). These data suggest that quiescent PSCs in human tissue are initially activated in response to culture conditions, a phenomenon that has been previously described in PSCs as well as hepatic stellate cells. ${ }^{9,17}$ Taken together, primary PSCs isolated from normal pancreatic specimens were qualitatively indistinguishable from pancreatitis- and PC-derived PSCs in culture.

Flow cytometric analysis was then performed to further quantify cellular phenotypes. Population distributions revealed that $>95 \%$ of cells in each stromal outgrowth expressed $\alpha$-SMA with a subset expressing GFAP, consistent with current descriptions of PSC biology (Figure 2b). ${ }^{10}$ Less than $5 \%$ of stellate cell outgrowths demonstrated weak ESA positivity. Importantly, this pattern was not observed in commercially available HPF, which demonstrated $<1 \%$ $\alpha$-SMA-positivity and a higher proportion of ESA-positive cells. To determine if the ESA-positive population in primary outgrowths represented contaminating epithelial cells, this population was isolated by flow cytometric cell sorting and subsequently subcultured. The subcultures were phenotypically similar to the primary culture; these cells had stellate morphology and subsequently stained positive for $\alpha$-SMA (Supplementary Figure 1). Taken together, these data quantitatively confirm that primary pancreatic stromal outgrowths demonstrate phenotypes consistent with activated PSCs, lack epithelial contamination, and are in contrast with the observed phenotype of commercially available HPF.

\section{Characteristic Phenotypes of PSCs in Culture}

PSC outgrowth cultures demonstrated exponential growth in culture with limited proliferative capacity, consistently undergoing senescence between passages 8 and 10 (Figure 3a). Primary PSC cultures generated from normal pancreatic tissue (NAS) appeared to have less replicative potential with respect to CP (IAS) and PC (TAS), achieving average population doublings of $7.8 \mathrm{vs} 10.3$ and 10.1, respectively $(P<0.05)$.

Vitamin A-containing lipid droplets represent an established hallmark of quiescent PSCs, a property that enables their isolation using a single density gradient method as described by Apte et al. ${ }^{12}$ However, upon culture-induced activation, demonstrated by universal $\alpha$-SMA expression, PSCs lose their vitamin A-containing lipid droplets. In order to determine whether PSC outgrowths retain plasticity with respect to activation state, adipocyte differentiation medium 
NAS
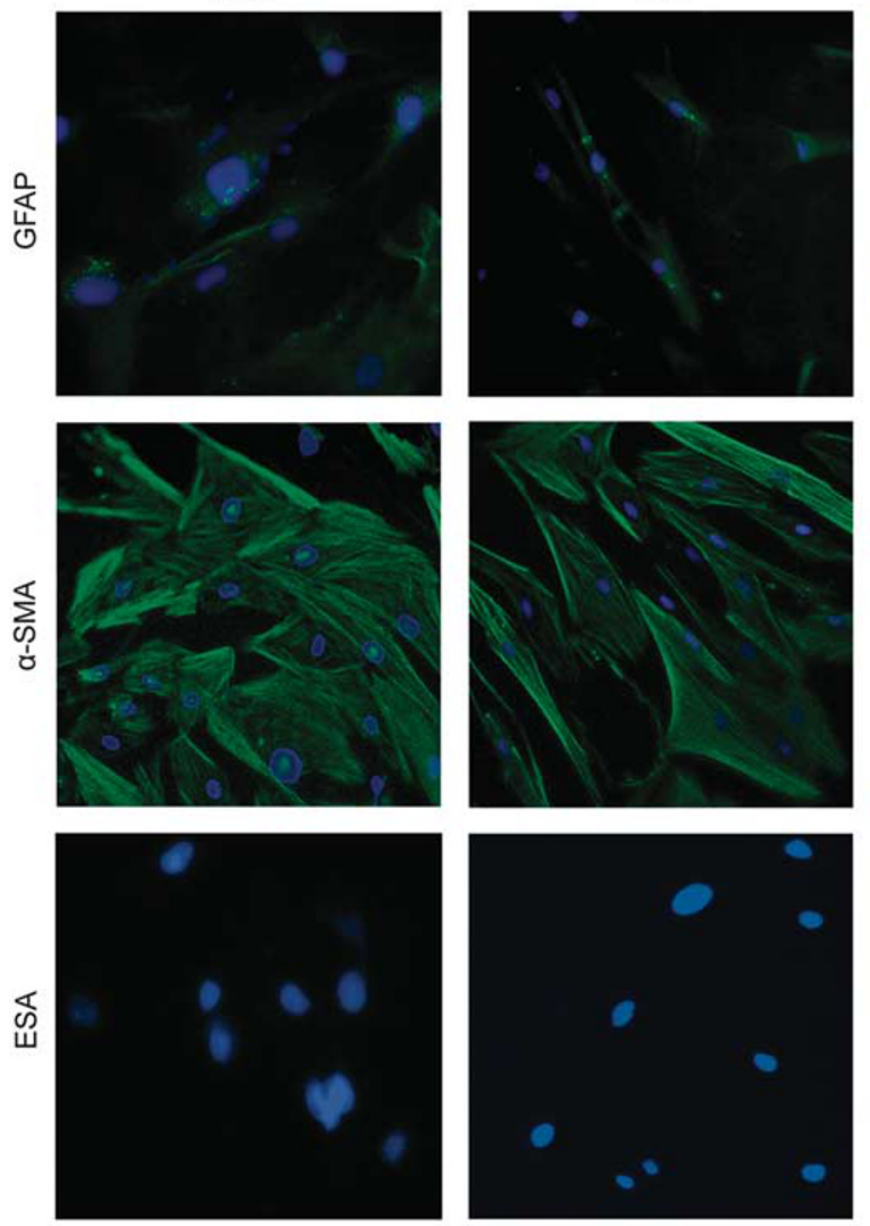

b
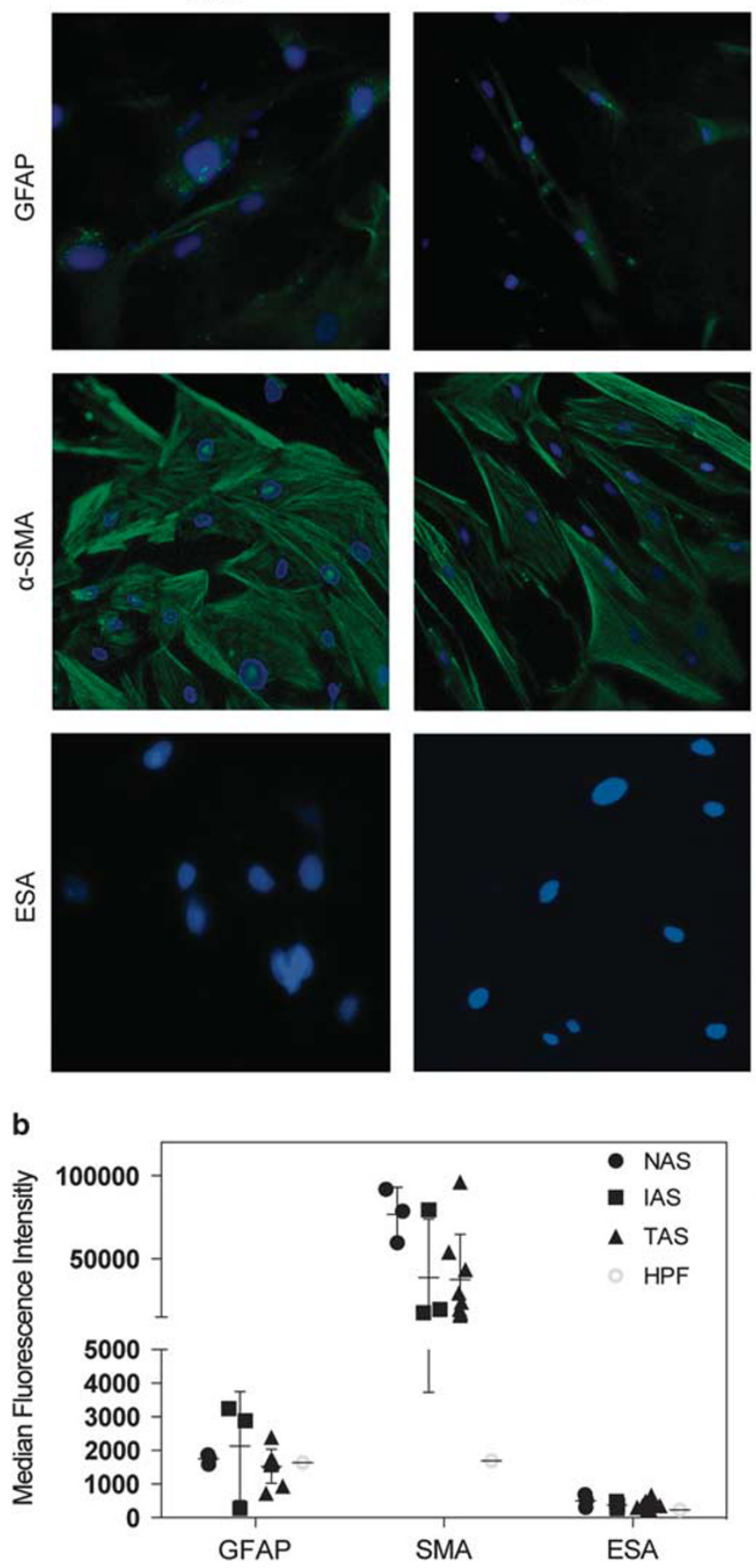

TAS
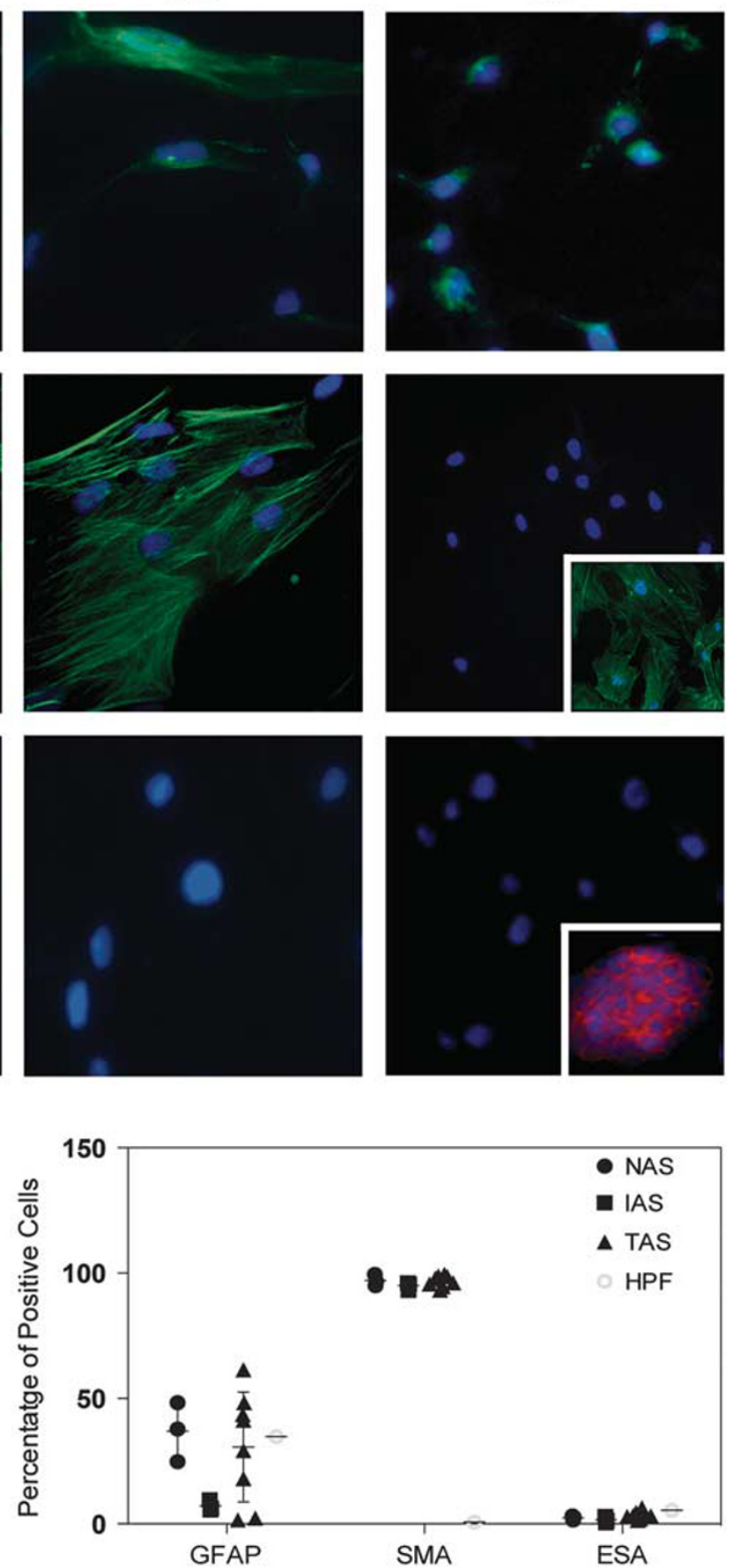

Figure 2 Expression of activated PSC markers in stromal outgrowths. (a) Representative immunofluorescent images demonstrate the expression of GFAP (green), $a$-SMA (green), ESA (red) and DAPI (blue) in fixed, permeabilized stromal outgrowths. HPF cells were stained using phalloidin to verify the presence of cytoskeletal structures (inset of middle panel) and ESA staining was performed on the PC cell line L3.6pl (inset of bottom panel) as a positive control. Cells are viewed at $\times 40$ magnification. (b) Flow cytometric analysis of $a$-SMA, GFAP, and ESA in pancreatic stromal outgrowths is displayed. DAPI, 4',6Diamidino-2-Phenylindole; ESA, epithelial surface antigen; GFAP, glial fibrillary acidic protein; HPF, human pancreatic fibroblast; IAS, inflammation-associated stroma; NAS, normal-associated stroma; PC, pancreatic cancer; PSC, pancreatic stellate cell; TAS, tumor-associated stroma; $a$-SMA, $a$-smooth muscle actin.

(ADM) was employed in an attempt to drive cultured PSCs into a quiescent phenotype. Indeed, ADM administration led to cellular shrinking and the accumulation of perinuclear droplets, confirmed by Oil Red $\mathrm{O}$ staining to be lipid containing stores (Figure $3 \mathrm{~b}$ ). In contrast to primary PSCs, the HPF cell line did not demonstrate these features despite ADM treatment. Culture-induced activation of PSCs therefore represents a potentially reversible process, suggesting the 
a

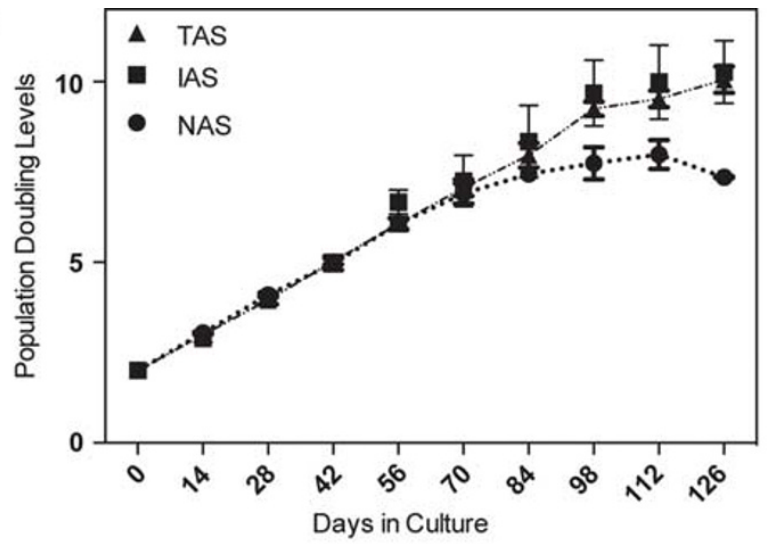

b
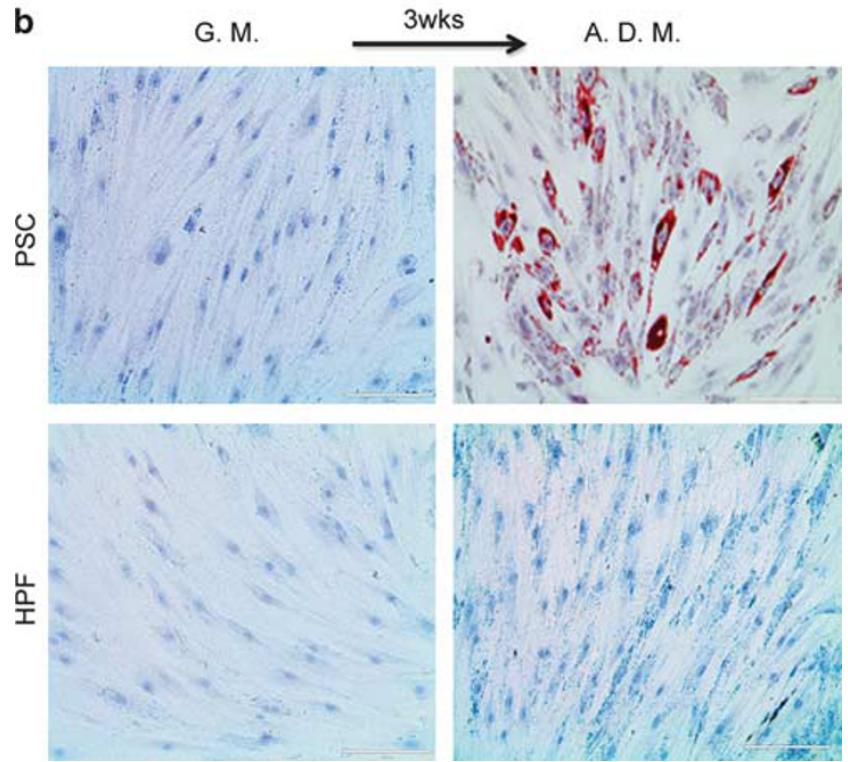

Figure 3 PSC replication and activation in culture. (a) Growth curves are displayed for primary PSC lines in culture. (b) The application of ADM to PSCs was assessed using Oil Red O staining, demonstrating intracellular lipid stores. Images were visualized using the EVOS imaging system $(\times 20$ objective). ADM, adipogenic differentiation medium; GM, growth medium; HPF, human pancreatic fibroblast; IAS, inflammation-associated stroma; NAS, normal-associated stroma; PSC, pancreatic stellate cell; TAS, tumorassociated stroma.

maintenance of a certain extent of plasticity in primary cultured PSCs and modification of media conditions may prove an attractive method for the in vitro study of quiescent PSC biology.

\section{Secretory Profiles of PSCs Are Independent of Donor Pancreatic Pathology}

Emerging evidence implicates PSC activation in the pathogenesis of pancreatic disease states. ${ }^{18,19}$ However, the relationship between donor pancreatic pathology and PSC outgrowth biology remains unclear. Since the role of PSCs in pancreatic disease pathogenesis is often examined in the context of paracrine signaling, particularly with respect to inflammation, PSC biology was defined by soluble mediator secretory profiles in culture. Specifically, a panel of 41 secreted proteins including growth factors, chemokines, and cytokines were obtained from supernatants of representative NAS, IAS, and TAS outgrowths (Supplementary Table 1). These data demonstrate that PSC secretory profiles in culture do not differ significantly with respect to donor pancreatic pathology.

\section{Secretory Profiles Change Over Time in Culture}

We anticipated that PSC behavior evolves over time in culture, necessitating caution in the interpretation of experiments employing highly passaged PSCs. Indeed, PSC cultures demonstrated increased $\gamma$-H2AX expression coupled with decreased Ki-67 expression over time in culture (Figure 4b). This pattern is consistent with the evolution of primary cells in culture, exhibiting limited replicative potential and eventually undergoing senescence. ${ }^{20}$ Further, soluble mediator secretion profiles also evolved over time in culture. Interestingly, a predictable pattern emerged in that several cytokines, chemokines, and growth factors were secreted at higher concentrations in highly passaged PSCs (Figure 4c). Specifically, concentrations of IL-6, IL-8, GRO, MCP-1, FGF-2, Fractalkine, IFN- $\alpha 2$, and MCP-3 were significantly elevated in late passages of multiple PSC outgrowth cultures, prompting caution when employing later passages for experimental use.

\section{Co-Culture of PSCs with PC Cells Led to Consistent Changes in Growth Factor, Cytokine, and Chemokine Secretion}

Emerging evidence suggests the importance of paracrine signaling between PC cells and PSCs in cancer progression. ${ }^{21,22}$ In order to evaluate the impact of paracrine communication between PSCs and PC cells, supernatants of PSC/PC co-culture experiments were analyzed. Primary PSC outgrowths were co-cultured with PC cell lines in a 10:1 ratio to emphasize the PSC contribution to the secretory profile. Co-culture in this manner led to increased concentrations of several growth factors, cytokines, and chemokines frequently implicated in pancreatic disease pathogenesis, including but not limited to FGF-2, G-CSF, IL-8, and RANTES (Figure 5 and Supplementary Table S1). Importantly, no significant differences in soluble mediator profiles were observed between co-cultures employing different PC cell lines. Collectively, these data support the presence of complex interactions between primary PSCs and PC cells that can alter the pancreatic microenvironment during disease.

\section{PSCs Augment Growth and Metastasis of PC Cells}

In order to determine the functional significance of primary PSCs in PC engraftment and progression, we performed co-inoculations of tumor-associated PSCs with luciferasetransfected PC cells (L3.6pl-Luc). At an injection ratio of 1:100 L3.6pl-Luc PC cells to TAS cells, respectively, tumor growth was significantly augmented, as assessed using bioluminescence and tumor volume measurements (Figure 6a). Further, in contrast to the central necrosis observed in control tumors, H\&E sections of tumors from co-inoculated 

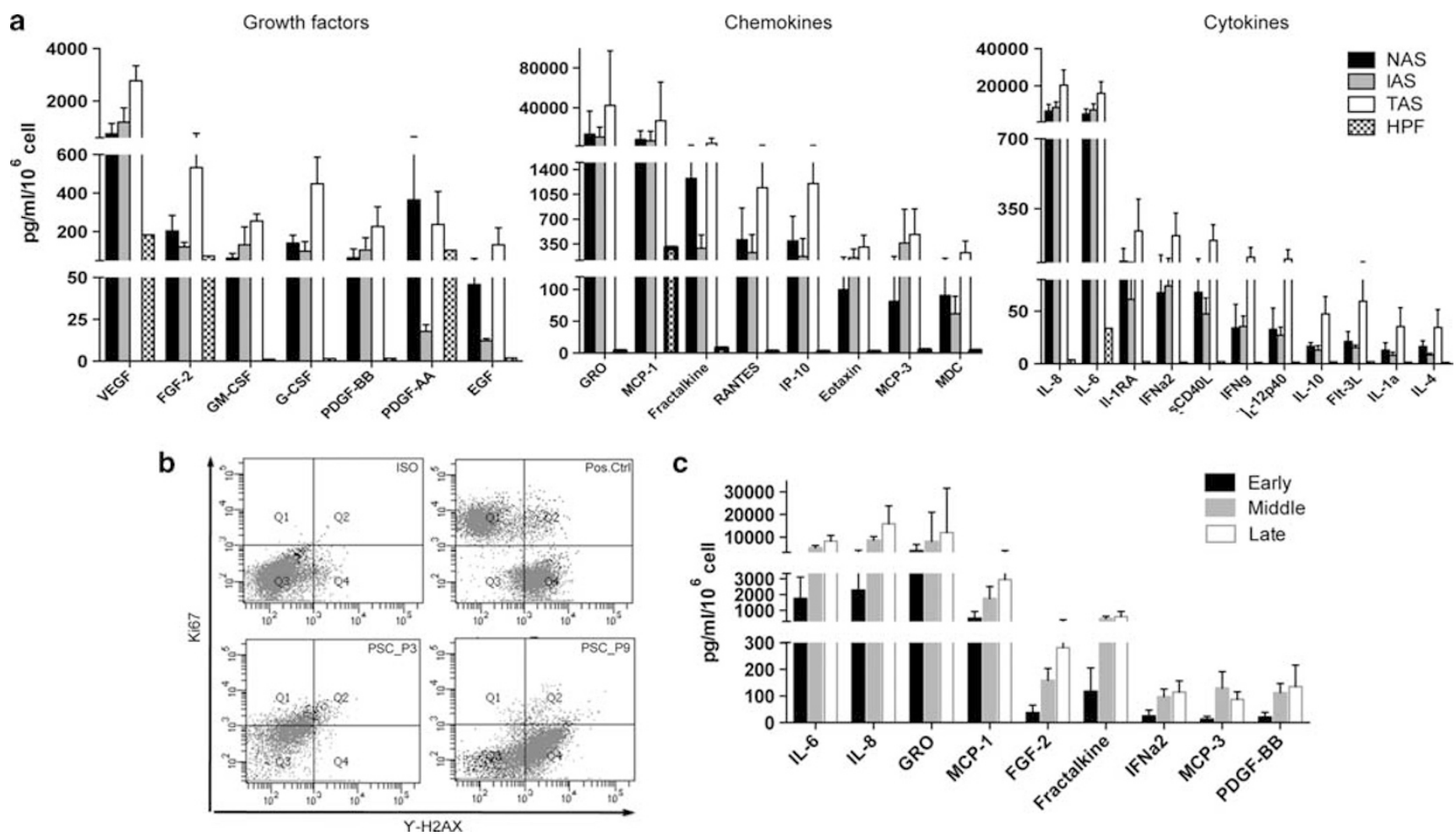

Figure 4 Soluble mediator secretion profiles of PSCs in culture. (a) NAS $(n=3)$, IAS $(n=3)$ and TAS $(n=6)$ cultures were serum starved for $24 \mathrm{~h}$ and supernatants were probed for the secretion of 41 growth factors, cytokines, and chemokines. (b) Flow cytometric analysis employing dual staining for Ki-67 and $\gamma$-H2AX. ISO: isotype control; Pos. Ctrl: Heat-induced senescent cells as positive control for assay; TAS_P3 and TAS_P9 represent PSCs in early passage (3) and late passage (9), respectively. (c) Evolution of TAS soluble mediator secretion profiles is displayed over time in culture. Supernatants from TAS cultures in early (1-3), middle (4-6), and late (7-9) passages were analyzed. Soluble mediator concentrations were normalized to cell number and presented as $\mathrm{pg} / \mathrm{ml} / 10^{6}$ cells. Bars represent mean \pm s.e.m. Statistical significance was determined using the Kruskal-Wallis test. HPF, human pancreatic fibroblast; IAS, inflammation-associated stroma; NAS, normal-associated stroma; PSC, pancreatic stellate cell; TAS, tumor-associated stroma.

mice revealed a desmoplastic-rich architecture with pockets of viable areas centrally (Figure 6b). Immediate bioluminescent imaging of explanted lungs demonstrated pulmonary metastasis in three of four (75\%) PSC co-inoculated mice, which was additionally confirmed with H\&E analysis (Figure 6c). Metastatic lesions were absent in control mice. These results suggest a supportive, functional role of primary PSCs in the PC microenvironment.

\section{DISCUSSION}

The capacity to isolate and culture PSCs initiated a surge of research in pancreatic stromal biology, particularly in the context of the PC microenvironment. Limitations in the availability of pancreatic surgical specimens gave rise to the immortalization of PSCs. ${ }^{23}$ Transformed PSC lines provided the additional benefit of eliminating potential confounders, such as variability between PSC isolation and culture practices. However, immortalization has been met with skepticism in the most recent PSC consensus conference. ${ }^{10}$ In part, this work serves to validate those concerns and help standardize the approach to primary PSC isolation for in vitro and in vivo experimentation. Regardless of PSC donor pathology, we demonstrate consistent outgrowth phenotypes with respect to growth properties, onset of senescence, and expression of previously validated biomarkers. This consistency is further confirmed on analysis of soluble mediator secretion, suggesting insignificant differences in PSC outgrowth behavior with respect to donor pathology. We demonstrate that secretion patterns remain dynamic over time in culture, providing scientific support for the generally accepted practice of excluding highly passaged primary cells from investigation. Finally, consistent with previously published data incorporating PSCs into PC xenograft models, we demonstrate augmentation of tumor growth and metastasis upon co-inoculation with PC cells.

Our data is consistent with previous reports exploring the functional aspects of PSCs in the PC microenvironment. ${ }^{13,24}$ We found an augmentation in tumor growth from the supplementation of PC cells with activated PSCs, and this finding is consistent with models incorporating similar primary PSC outgrowth cultures. ${ }^{13,25-27}$ Also consistent with our data, others have previously demonstrated augmented metastasis of tumors upon co-inoculation of PSCs with PC cells in murine xenograft models. ${ }^{28}$ In contrast, stromal depletion strategies incorporated into genetically engineered mouse models of PC have recently provided data in 
a
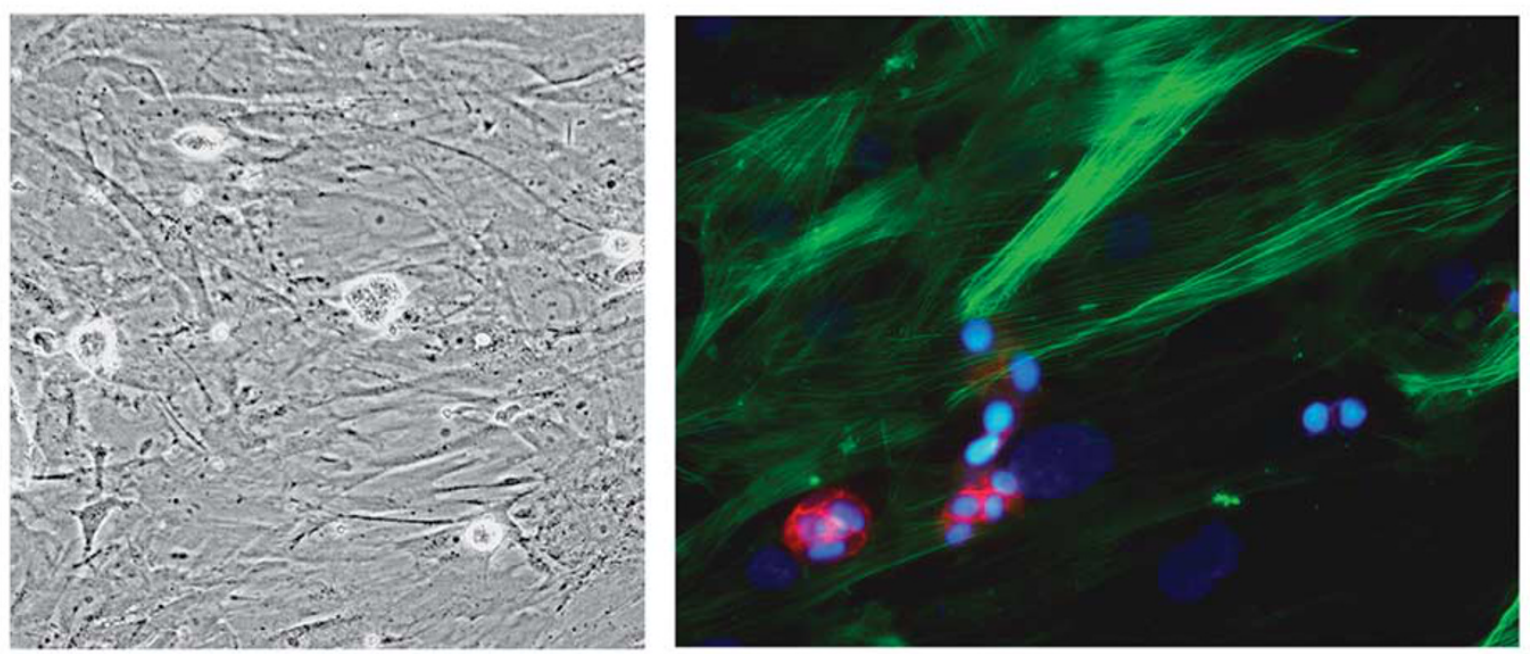

b

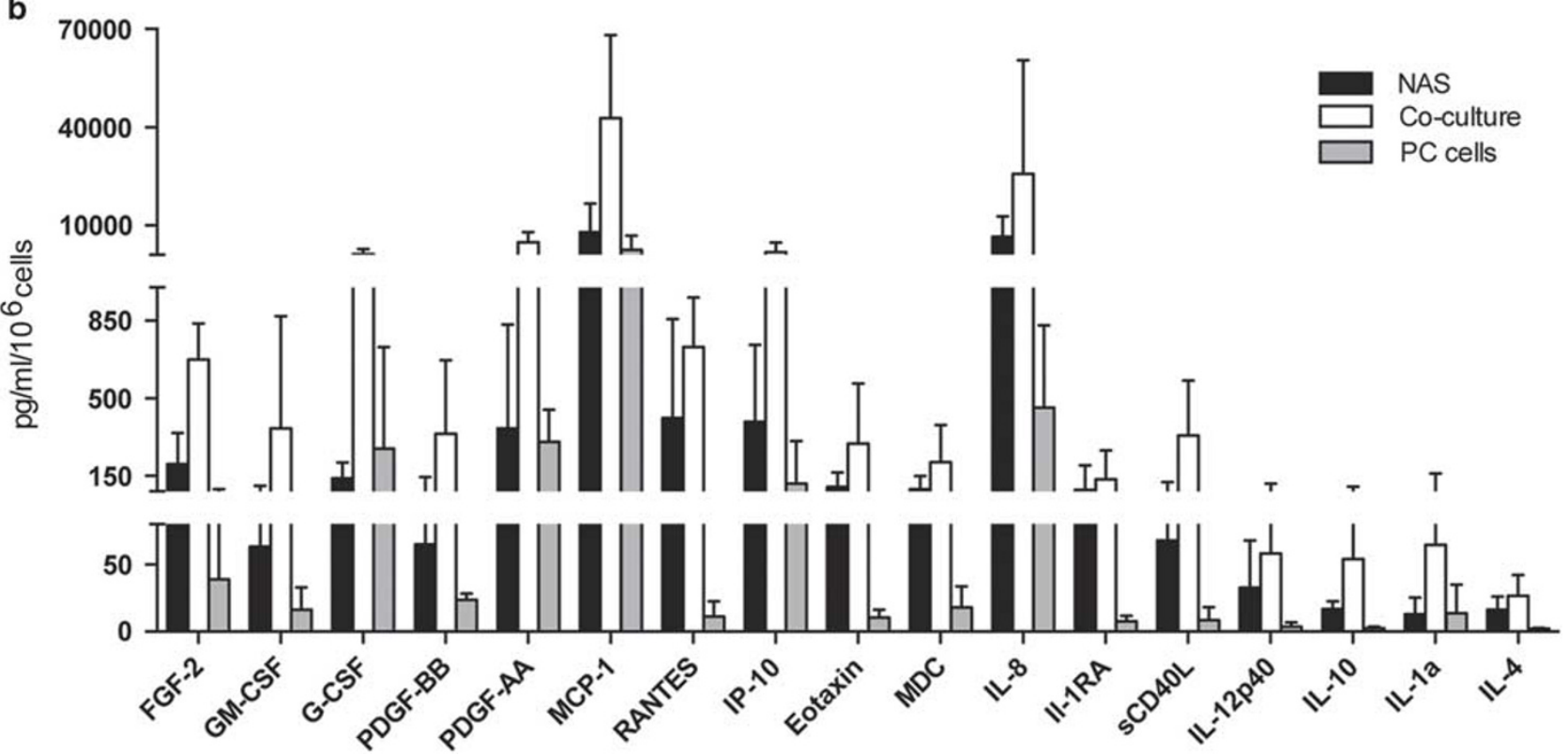

Figure 5 Growth factor, cytokine, and chemokine secretion profiles of PSC/PC co-cultures. (a) PSCs were co-cultured with PC cells at a 10:1 ratio, demonstrated by phase-contrast image $(\times 10)$ and immunofluorescent staining with ESA (red), $a$-SMA (green) and DAPI (blue). (b) PSCs ( $n=3)$ represent three early passage (P1-3) NAS outgrowth lines. Co-cultures represent three early passage NAS outgrowths in combination with the PC cell lines L3.6pl, PANC-1, and BxPC3. Each possible NAS/PC cell pair was co-cultured ( $n=9$ total). Soluble mediator concentrations were normalized to cell number and presented as pg/ml $/ 10^{6}$ cells. Bars represent mean+s.e.m. for each aggregate cell type. Co-culture bars (white) represent the mean+s.e.m. of nine coculture pairs. Statistical analysis employed the Mann-Whitney U-test between PSCs alone and co-cultures. DAPI, 4',6-Diamidino-2-Phenylindole; ESA, epithelial surface antigen; NAS, normal-associated stroma; PC, pancreatic cancer; PSC, pancreatic stellate cell; $a$-SMA, $a$-smooth muscle actin.

opposition of this concept of activated PSCs supporting PC progression. ${ }^{5,6}$ However, it is also important to note that the modulation of PSC activation, rather than its elimination via prevention or conditional lethality, alleviated PC progression in work employing similar genetically engineered mouse models in PC. ${ }^{9}$ This modulation suggests a contextdependent tumor-promoting role of activated PSCs. Our work therefore builds on similar investigations into PSC biology and expands upon current knowledge regarding human PSC isolation and functional characterization that will continue to be a vital consideration for further investigation into PC tumor-stromal interactions.

The lack of variability in marker expression and secretion patterns with respect to donor pathology challenges the concept of a unique TAS phenotype. Erkan et al. ${ }^{29}$ recently performed comprehensive gene expression profiles of PSC outgrowths from pancreatitis, PC, PC liver metastases and cirrhotic livers. Similarly, Pilarsky et al. ${ }^{30}$ performed tissue microdissection of pancreatitis and PC samples and subsequent gene expression of stromal elements. Each of these 

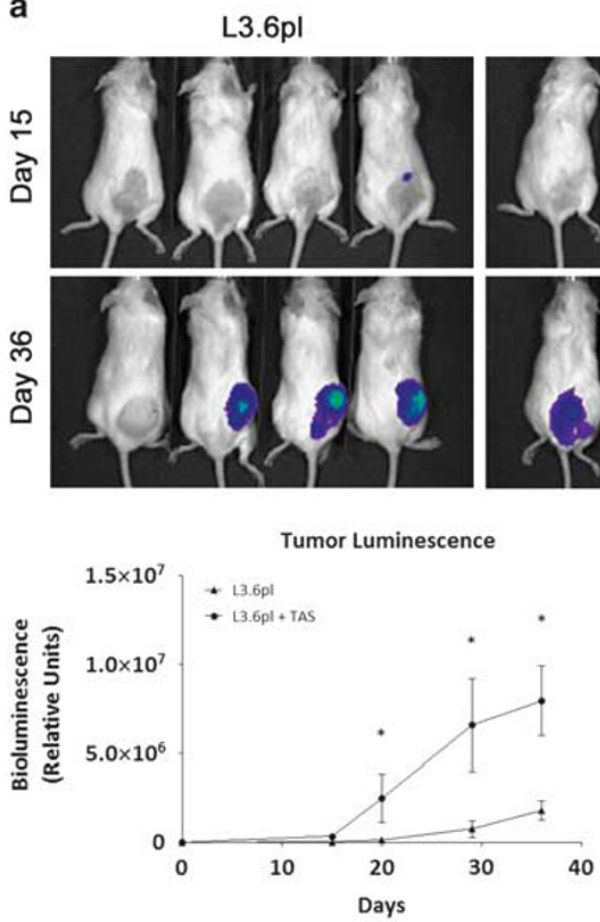

L3.6pl + TAS

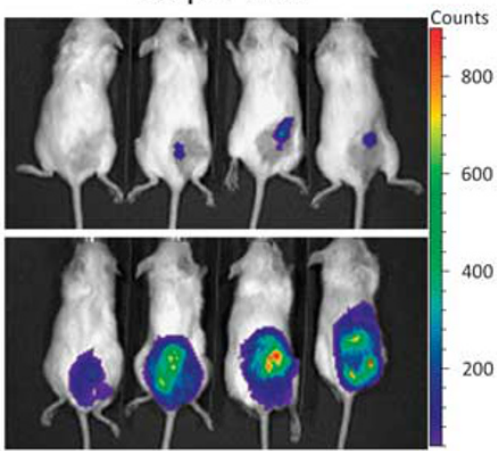

Tumor Volume

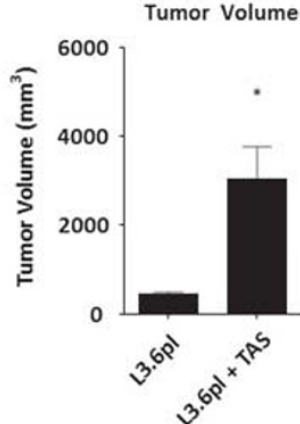

b
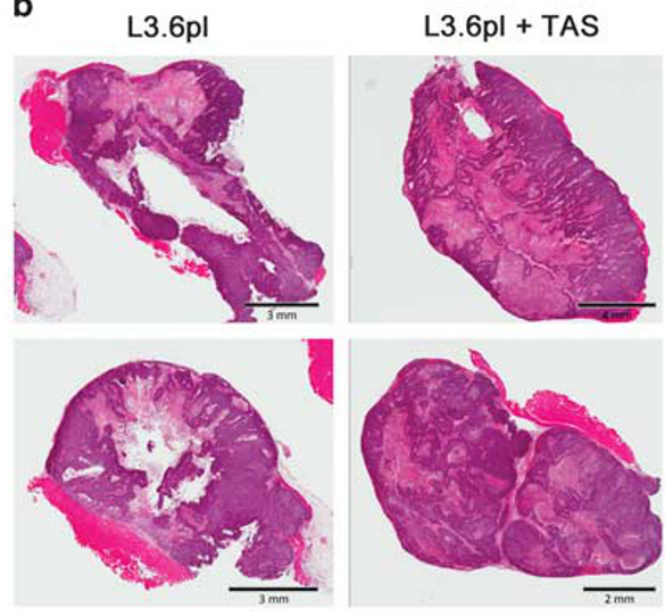

C

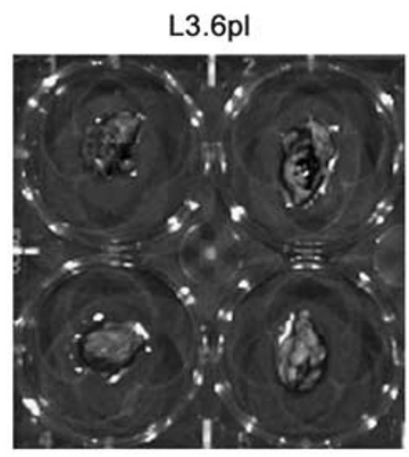

L3.6pl + TAS

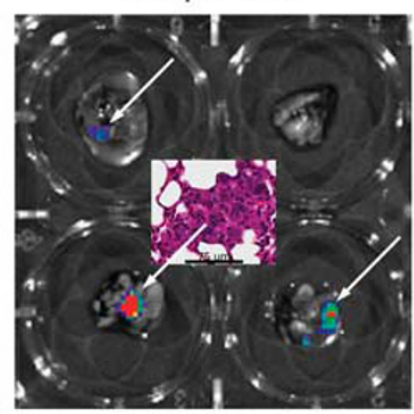

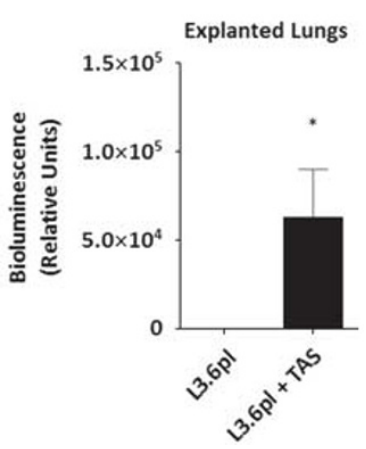

Figure 6 Co-inoculation of PSCs with PCS in a murine xenograft model. TAS cells were co-injected with L3.6pl cells at a 100:1 ratio, respectively, into NOD-SCID mice. (a) Bioluminescent imaging on post-implantation days 15 and 36 are displayed as well as tumor volumes upon explanation. Data are presented as mean \pm s.e.m. (b) Whole tissue xenografts were paraffin-embedded and examined by H\&E staining. (c) Bioluminescent imaging of explanted murine lungs and representative H\&E staining (inset) of pulmonary metastatic lesions are shown. Scale bars indicate magnification for micrographs. Groups were compared using the Mann-Whitney U-test. ${ }^{*} P<0.05$. H\&E, hematoxylin and eosin; PC, pancreatic cancer; PSC, pancreatic stellate cell; TAS, tumor-associated stroma.

investigations reached the conclusion that stellate cell expression patterns differed between cancer and benign inflammation. Importantly, co-injection models have demonstrated that PSCs derived from nonmalignant pancreatic specimens augment PC growth and metastasis in a manner similar to TAS. ${ }^{28}$ Regardless of function, genes implicated as cancer specific by these investigations were not analyzed in our experiments. We instead chose to focus on soluble mediators that may directly involve pathogenic paracrine signaling pathways, particularly in the development and maintenance of a chronic inflammatory state. Despite these identified differences in mRNA expression based on the underlying pathology from which these cells are isolated, our data would suggest that PSCs derived from malignant tissues should not be excluded as a potentially useful model for in vitro studies of human PSC biology in non-malignant disease states.

In summary, we present data that suggest that isolation and expansion of human PSC using the outgrowth methodology from a diverse array of donor pathologies may serve as a source of primary cultures of activated and quiescent PSCs for in vitro and in vivo experimentation. This work provides a 
foundation for further investigation into the role of PSCs in the pathogenesis of pancreatic disease with enormous translational value.

Supplementary Information accompanies the paper on the Laboratory Investigation website (http://www.laboratoryinvestigation.org)

\section{ACKNOWLEDGMENTS}

This work was supported by NCI 5T32CA106493-09, Cracchiolo Foundation.

\section{DISCLOSURE/CONFLICT OF INTEREST}

The authors declare no conflict of interest.

1. Ceyhan GO, Friess H. Pancreatic disease in 2014: Pancreatic fibrosis and standard diagnostics. Nat Rev Gastroenterol Hepatol 2015;12:68-70.

2. Erkan M, Hausmann S, Michalski CW et al. The role of stroma in pancreatic cancer: diagnostic and therapeutic implications. Nat Rev Gastroenterol Hepatol 2012;9:454-467.

3. Vonlaufen A, Joshi S, Qu C et al. Pancreatic stellate cells: partners in crime with pancreatic cancer cells. Cancer Res 2008;68:2085-2093.

4. Apte MV, Haber PS, Darby SJ et al. Pancreatic stellate cells are activated by proinflammatory cytokines: implications for pancreatic fibrogenesis. Gut 1999;44:534-541.

5. Rhim $A D$, Oberstein $P E$, Thomas $\mathrm{DH}$ et al. Stromal elements act to restrain, rather than support, pancreatic ductal adenocarcinoma. Cancer Cell 2014;25:735-747.

6. Ozdemir BC, Pentcheva-Hoang T, Carstens JL et al. Depletion of carcinoma-associated fibroblasts and fibrosis induces immunosuppression and accelerates pancreas cancer with reduced survival. Cancer Cell 2014;25:719-734.

7. Lonardo E, Frias-Aldeguer J, Hermann PC et al. Pancreatic stellate cells form a niche for cancer stem cells and promote their self-renewal and invasiveness. Cell Cycle 2012;11:1282-1290.

8. Ikenaga N, Ohuchida K, Mizumoto K et al. CD10+ pancreatic stellate cells enhance the progression of pancreatic cancer. Gastroenterology 2010;139:1041-1051.

9. Sherman $\mathrm{MH}, \mathrm{Yu} \mathrm{RT}$, Engle DD et al. Vitamin d receptor-mediated stromal reprogramming suppresses pancreatitis and enhances pancreatic cancer therapy. Cell 2014;159:80-93.

10. Erkan M, Adler G, Apte MV et al. StellaTUM: current consensus and discussion on pancreatic stellate cell research. Gut 2012;61:172-178.

11. Bachem MG, Schneider E, Gross $\mathrm{H}$ et al. Identification, culture, and characterization of pancreatic stellate cells in rats and humans. Gastroenterology 1998;115:421-432.

12. Apte MV, Haber PS, Applegate TL et al. Periacinar stellate shaped cells in rat pancreas: identification, isolation, and culture. Gut 1998;43:128-133.

13. Hwang RF, Moore $\mathrm{T}$, Arumugam $\mathrm{T}$ et al. Cancer-associated stromal fibroblasts promote pancreatic tumor progression. Cancer Res 2008;68: 918-926.
14. Bruns CJ, Harbison MT, Kuniyasu $\mathrm{H}$ et al. In vivo selection and characterization of metastatic variants from human pancreatic adenocarcinoma by using orthotopic implantation in nude mice. Neoplasia 1999;1:50-62.

15. Trevino JG, Summy JM, Gray MJ et al. Expression and activity of SRC regulate interleukin-8 expression in pancreatic adenocarcinoma cells: implications for angiogenesis. Cancer Res 2005;65:7214-7222.

16. Choi YS, Cha SM, Lee YY et al. Adipogenic differentiation of adipose tissue derived adult stem cells in nude mouse. Biochem Biophys Res Commun 2006;345:631-637.

17. Hellerbrand C, Jobin C, Licato LL et al. Cytokines induce NF-kappaB in activated but not in quiescent rat hepatic stellate cells. Am J Physiol 1998;275(2 Pt 1):G269-G278.

18. Bachem MG, Zhou S, Buck K et al. Pancreatic stellate cells-role in pancreas cancer. Langenbecks Arch Surg 2008;393:891-900.

19. Omary MB, Lugea A, Lowe AW et al. The pancreatic stellate cell: a star on the rise in pancreatic diseases. J Clin Invest 2007;117:50-59.

20. Lawless C, Wang C, Jurk D et al. Quantitative assessment of markers for cell senescence. Exp Gerontol 2010;45:772-778.

21. Paulo JA, Urrutia R, Banks PA et al. Proteomic analysis of an immortalized mouse pancreatic stellate cell line identifies differentially-expressed proteins in activated vs nonproliferating cell states. J Proteome Res 2011;10:4835-4844.

22. Yoshida $S$, Yokota $T$, Ujiki $M$ et al. Pancreatic cancer stimulates pancreatic stellate cell proliferation and TIMP-1 production through the MAP kinase pathway. Biochem Biophys Res Commun 2004;323: $1241-1245$.

23. Jesnowski R, Furst D, Ringel J et al. Immortalization of pancreatic stellate cells as an in vitro model of pancreatic fibrosis: deactivation is induced by matrigel and N-acetylcysteine. Lab Invest 2005;85: 1276-1291.

24. Fujita $\mathrm{H}$, Ohuchida $\mathrm{K}$, Mizumoto $\mathrm{K}$ et al. Tumor-stromal interactions with direct cell contacts enhance proliferation of human pancreatic carcinoma cells. Cancer Sci 2009;100:2309-2317.

25. Bachem MG, Schunemann M, Ramadani $M$ et al. Pancreatic carcinoma cells induce fibrosis by stimulating proliferation and matrix synthesis of stellate cells. Gastroenterology 2005;128:907-921.

26. Erkan M, Kleeff J, Gorbachevski $A$ et al. Periostin creates a tumorsupportive microenvironment in the pancreas by sustaining fibrogenic stellate cell activity. Gastroenterology 2007;132:1447-1464.

27. Schneiderhan W, Diaz F, Fundel M et al. Pancreatic stellate cells are an important source of MMP-2 in human pancreatic cancer and accelerate tumor progression in a murine xenograft model and CAM assay. J Cell Sci 2007;120(Pt 3):512-519.

28. Xu Z, Vonlaufen A, Phillips PA et al. Role of pancreatic stellate cells in pancreatic cancer metastasis. Am J Pathol 2010;177:2585-2596.

29. Erkan M, Weis N, Pan Z et al. Organ-, inflammation- and cancer specific transcriptional fingerprints of pancreatic and hepatic stellate cells. Mol Cancer 2010;9:88.

30. Pilarsky C, Ammerpohl O, Sipos B et al. Activation of Wnt signalling in stroma from pancreatic cancer identified by gene expression profiling. J Cell Mol Med 2008;12(6B):2823-2835. 